\title{
Is IR an Essential Hospital Service? Analysis of Trauma Procedures at a Level 1 Centre During the First Wave of COVID- 19 Pandemic in Australia
}

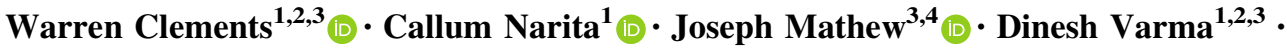 \\ Mark C. Fitzgerald ${ }^{3,4}$ (ID) Gerard S. Goh ${ }^{1,2,3}$ (ID)
}

Received: 27 August 2020/Accepted: 19 September 2020/Published online: 9 October 2020

(C) Springer Science+Business Media, LLC, part of Springer Nature and the Cardiovascular and Interventional Radiological Society of Europe (CIRSE) 2020

To the editor

The World Health Organization (WHO) declared SARS-CoV-2 (COVID-19) a global pandemic on 12 March 2020 [1]. This led to limitation of non-essential hospital services and in some circumstances, recruitment of specialty services into front-line critical care services [2]. Many interventional radiologists (IRs) modified their service to provide alternative procedures to patients affected by COVID-19 but stressed the importance of protecting IR services for their hospital [3].

Like other countries, Australia adopted early strict social distancing measures. Local government declared a state of emergency on 16 March 2020 and recommended that

Warren Clements

W.clements@alfred.org.au

Callum Narita

callumnarita@gmail.com

Joseph Mathew

j.mathew@alfred.org.au

Dinesh Varma

d.varma@alfred.org.au

Mark C. Fitzgerald

m.fitzgerald@alfred.org.au

Gerard S. Goh

G.goh@alfred.org.au

1 Department of Radiology, Alfred Health, 55 Commercial Road, Melbourne, VIC 3004, Australia

2 Department of Surgery, Monash University Central Clinical School, Melbourne, Australia

3 National Trauma Research Institute, Monash University Central Clinical School, Melbourne, Australia

4 Department of Trauma, Alfred Health, Melbourne, Australia people stay at home unless necessary [1]. Many businesses closed or were mandated to work from home. Schools also transitioned to online learning. There was an associated decrease in road traffic [4]. On 31 May 2020, the state government lifted initial restrictions on social distancing.

Melbourne has a population of approximately 5 million. As a level 1 trauma centre, the Alfred Hospital is the largest of 2 trauma centres in the state. This study aimed to quantify the number and acuity of IR procedures performed on trauma patients during the first wave.

Local institutional review board approval was obtained. The study covered the period from declaration of a state of emergency on 16 March 2020 to the lifting of social restrictions on 31 May 2020, also known as the "first wave". The hospital Radiology Information System and the Alfred trauma database were searched for trauma inpatients who received an IR procedure during the above period. The same data were then gathered for the corresponding dates in 2019 (16 March 2019-31 May 2019) for direct comparison.

During the first wave, there were 40 procedures performed on 28 trauma patients (Fig. 1) with a mean age of $53.7 \pm 19.2$ years, of which $75 \%$ were male, and the median ISS was 29 (range 8-50). This compares with 32 procedures during the same dates in 2019 on 25 trauma patients with a mean age of $48.7 \pm 18.1$ years, of which $62.5 \%$ were male, with a median ISS of 29 (range 1-66). This constituted an overall $25 \%$ increase. There were no significant differences in the mean age, gender, or ISS between the groups (Table 1). In 2020, $22.5 \%$ of procedures were performed afterhours compared with $21.9 \%$ in 2019. There was also a similar distribution of the overall acuity of procedures (Table 2), type of procedure, and mechanism of injury. 
Fig. 1 Mechanism of injury for trauma IR procedures during 2020 COVID-19 first wave

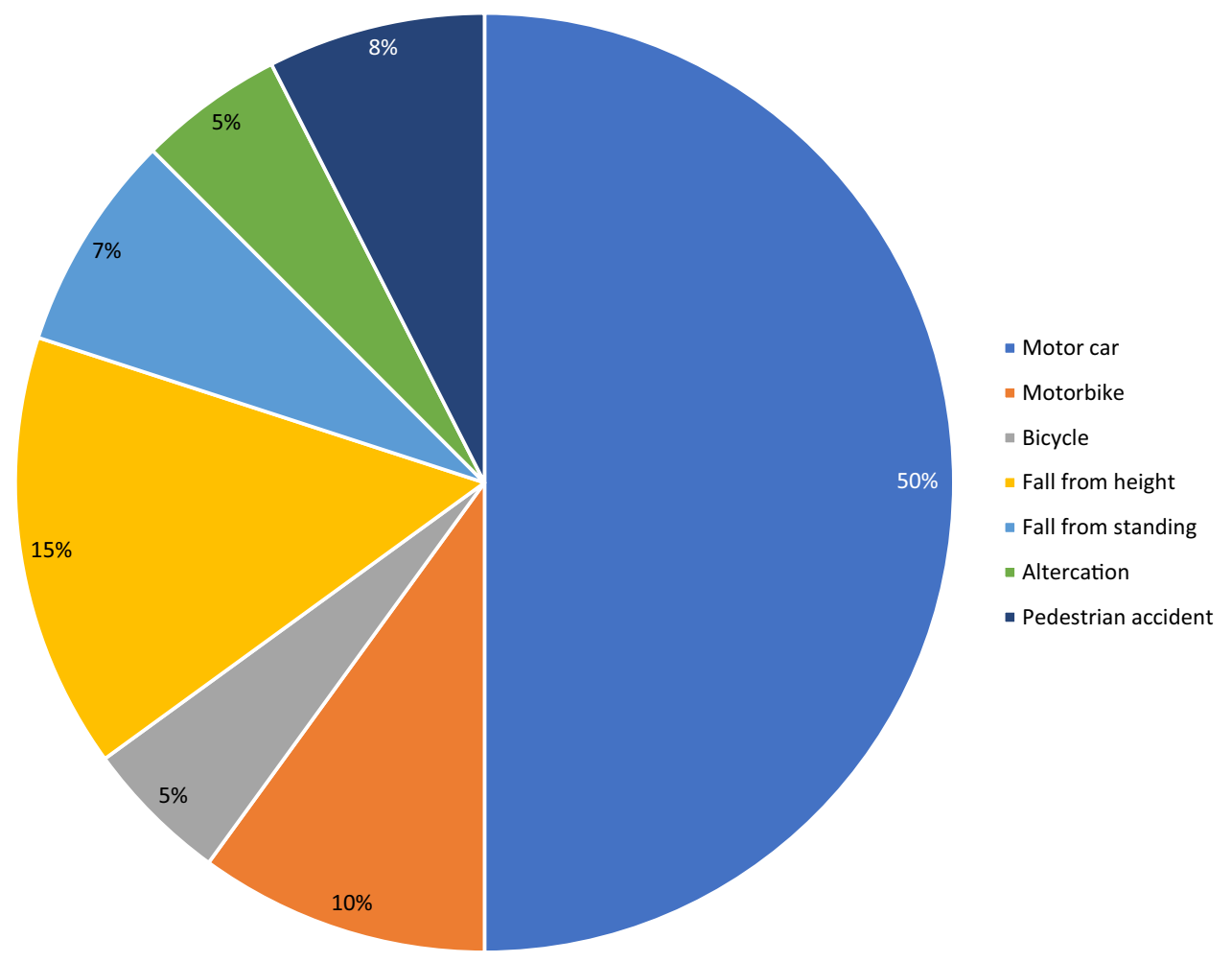

\begin{tabular}{lll}
\hline & 2020 first wave & 2019 corresponding dates \\
\hline Age (mean, SD) & $53.7 \pm 19.2$ & $48.8 \pm 18.1$ \\
Male gender (number, \%) & $30,75 \%$ & $20,62.5 \%$ \\
ISS (median, range) & $29(8-50)$ & $29(1-66)$ \\
During business hours (number, \%) & $31,77.5 \%$ & $25,78.1 \%$ \\
After hours (number, \%) & $9,22.5 \%$ & $7,21.9 \%$
\end{tabular}

All groups, $p>0.05$
Table 2 Categorisation of procedure urgency

\begin{tabular}{lll}
\hline & 2020 first wave & 2019 corresponding dates \\
\hline Emergent (number, \%) & $9,22.5 \%$ & $11,34.4 \%$ \\
Urgent (number, \%) & $24,60 \%$ & $14,43.75 \%$ \\
Routine (number, \%) & $7,17.5 \%$ & $7,21.9 \%$
\end{tabular}

All groups, $p>0.05$

During an international health crisis such as a global pandemic, hospital administrators will be placed in a challenging position of managing staff throughout their hospital to provide safety and care of all patients. As a part of this decision-making process, there must be a division of resources between what is considered essential and what is considered non-essential. However, there must remain essential resources which will still be required even during a lock-down period.

Some studies have shown that the overall workload in IR has suffered less from pandemic-related shutdown than for the total hospital workload [5]. This study showed that IR procedures performed on trauma patients during the first wave in Melbourne increased by $25 \%$. There are likely to be several reasons for this increase. While overall road traffic decreased during this time, travel to and from work was still permitted and local government building and road works increased to take advantage of the quieter roads, leading to heavy vehicle traffic. In addition, trauma patients continued to arrive from outside metropolitan Melbourne where social distancing restrictions were less strict. With more people not at work there was also an increase in pedestrian-related trauma.

IR services at a trauma hospital are critical to provide emergent or urgent care for patients, even during a 
government-mandated lockdown. Whilst all doctors must be flexible and provide patient care in any form when necessary, this study suggests that protection of IR services both in- and after-hours should be considered essential in the event of a "second wave" of COVID-19 or a future pandemic.

Acknowledgements The authors would like to acknowledge all staff in the Department of Radiology and Department of Trauma at the Alfred Hospital. In addition, the authors acknowledge the National Trauma Research Institute and the Alfred Trauma Registry.

Funding This manuscript was not supported by any funding.

\section{Compliance with Ethical Standards}

Conflict of interest The authors declare that they have no conflict of interest.

Ethical Approval All procedures performed in studies involving human participants were in accordance with the ethical standards of the institutional and/or national research committee and with the 1964 Helsinki Declaration and its later amendments or comparable ethical standards. All procedures performed in studies involving animals were in accordance with the ethical standards of the institution or practice at which the studies were conducted. In addition, institutional review board approval was obtained including a waiver of the need for consent in the retrospective study.

Consent for publication IRB provided a waiver of the need for consent for publication from individual participants in the study.
Informed Consent IRB provided a waiver of the need for consent from individual participants in the study.

\section{References}

1. Mikakos J.: Pandemic plan for the Victorian Health Sector. Victorian Department of Health and Human Services. https:// www2.health.vic.gov.au/ /media/Health/Files/Collections/ Research\%2520and\%2520reports/C/COVID-19-pandemic-plan. Accessed 15 Jul 2020.

2. Dunning J.: COVID-19 has taken me from surgeon to ICU nurse. https://rcni.com/nursing-standard/opinion/comment/covid-19-hastaken-me-surgeon-to-icu-nurse-159586. Accessed 15 Jul 2020.

3. Rostampour S, Cleveland $\mathrm{T}$, White $\mathrm{H}$, et al. Response of UK interventional radiologists to the COVID-19 pandemic-survey findings. CVIR Endovasc. 2020;3:41. https://doi.org/10.1186/ s42155-020-00133-2.

4. Nunez JH, Sallent A, Lakhani K, Guerra-Farfan E, Vidal N, Ekhtiari S, et al. Impact of the COVID-19 pandemic on an emergency traumatology service: experience at a tertiary trauma centre in Spain. Injury. 2020;51(7):1414-8.

5. Iezzi R, Valenta I, Cina A, et al. Longitudinal study of interventional radiology activity in a large metropolitan Italian tertiary care hospital how the COVID-19 pandemic emergency has changed our activity. Eur Radiol. 2020. https://doi.org/10.1007/ s00330-020-07041-y.

Publisher's Note Springer Nature remains neutral with regard to jurisdictional claims in published maps and institutional affiliations. 